\title{
Epithelioid angiomyolipoma of the liver: a case report
}

\author{
Soo Yeon Lee, and Baek-hui Kim \\ Department of Pathology, Korea University Guro Hospital, Korea University College of Medicine, Seoul, Korea
}

Epithelioid angiomyolipoma (EAML) of liver is a rare neoplasm. Hepatic EAML is often misdiagnosed as other neoplasms such as hepatocellular carcinoma due to non-specific clinical and radiologic features. The morphologic features under microscope and immunohistochemistry staining profile are important in the diagnosis EAML. Here, we report a case of 52-year-old man who found $1.2 \mathrm{~cm}$ mass in liver by routine checkup. On the impression of hepatocellular carcinoma, lateral sectionectomy of the liver was done. Microscopically, the tumor is composed of predominant epithelioid cells with vascular component and foamy cells. These cells were positive for HMB45, MelanA, and smooth muscle actin and negative for epithelial membrane antigen. The final diagnosis was hepatic EAML. (Clin Mol Hepatol 2017;23:91-94)

Keywords: Liver; Angiomyolipoma; Perivascular epithelioid cell neoplasms

\section{INTRODUCTION}

Angiomyolipoma (AML) is a benign mesenchymal tumor composed of perivascular epithelioid cells (PECS). There are some reports about association of $\mathrm{AML}$ and tuberous sclerosis complex, but most of AMLs were occurred sporadically. ${ }^{1,2}$ The most common site of AML occurrence is the kidney, but the liver is also a common site of $A M L$ occurrence. Epithelioid variant of $A M L$ (EAML) is characterized by predominance of epithelioid cells in the tumor components. It is known that EAMLs show more aggressive clinical behavior, and have more potential to undergo malignant transformation. ${ }^{1,3,4}$ Most EAML shows benign behavior, but some malignant EAMLs were reported in the literature. ${ }^{5,6}$ Hepatic EAML can mimic many other epithelioid hepatic tumors, such as hepatocellular carcinoma, and it is difficult to make precise diagnosis of EAML. ${ }^{5,7}$ In this report, we present a case of resected primary hepatic EAML and discuss about the characteristics of hepatic EAML.

\section{CASE REPORT}

A 52-year-old man who had no remarkable medical history had an abdominal sonography at local hospital for regular checkup. The scan revealed a $1.7 \mathrm{~cm}$ sized mass in the liver. He referred to our hospital for further evaluation. A computed tomography scan was performed. The mass measures $1.5 \mathrm{~cm}$ at segment 2 of liver. (Fig. 1A, B) It is enhanced on arterial phase, and washed out on delayed phase, suggestive of hepatocellular carcinoma. There was no other specific finding. The initial laboratory findings showed normal liver function tests: aspartate aminotransferase $23 \mathrm{IU} / \mathrm{L}$, alanine aminotransferase $19 \mathrm{IU} / \mathrm{L}$, gamma-glutamyl transpeptidase $25 \mathrm{IU} / \mathrm{L}$, and alkaline phosphatase $77 \mathrm{IU} / \mathrm{L}$, total bilirubin $0.33 \mathrm{mg} / \mathrm{dL}$, and direct bilirubin $0.14 \mathrm{mg} / \mathrm{dL}$. Tumor marker tests for carbohydrate antigen 19-9, carcinoembryonic antigen, alphafetoprotein were all within reference range. The tests for hepatitis $B$ and $C$ virus were negative. The impression for mass was hepa-

\section{Abbreviations:}

AML, angiomyolipoma; EAML, epithelioid angiomyolipoma; EMA, epithelial membrane antigen; $\mathrm{FNH}$, focal nodular hyperplasia; $\mathrm{HA}$, hepatocellular adenoma; HCC, hepatocellular carcinoma; PEC, perivascular epithelioid cell; SMA, smooth muscle actin

\section{Corresponding author: Baek-hui Kim}

Department of Pathology, Korea University Guro Hospital, Korea University College of Medicine, 148 Gurodong-ro, Guro-gu, Seoul 08308, Korea

Tel: +82-2-2626-3255, Fax: +82-2-2626-1486

E-mail:maelstrom@naver.com 


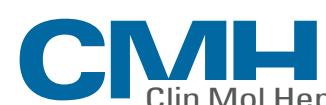

Clin Mol Hepatol

Volume 23 Number_1 March 2017
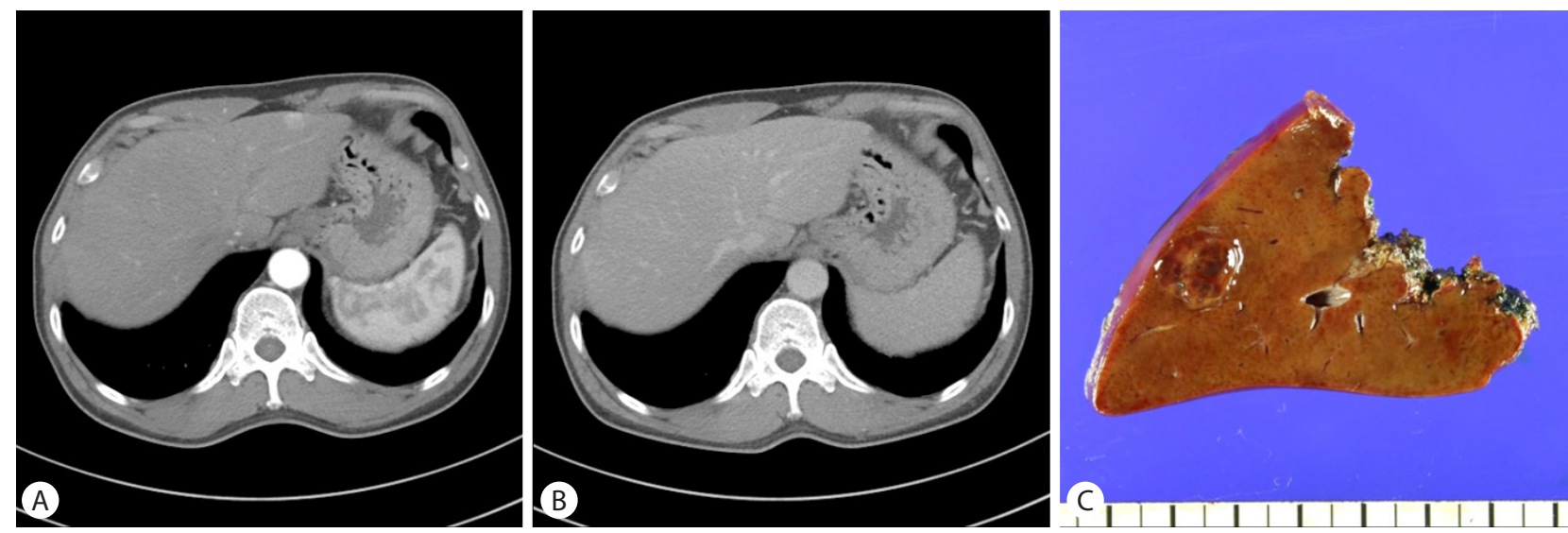

Figure 1. Imaging and gross pathologic findings of tumor. (A) Enhanced computed tomography imaging of arterial phase shows an enhanced mass at segment 2 of the liver. (B) Portal phase shows decreased enhancement of mass. (C) The cut surface of lateral sectionectomy specimen of liver shows tan colored round well defined nodule.
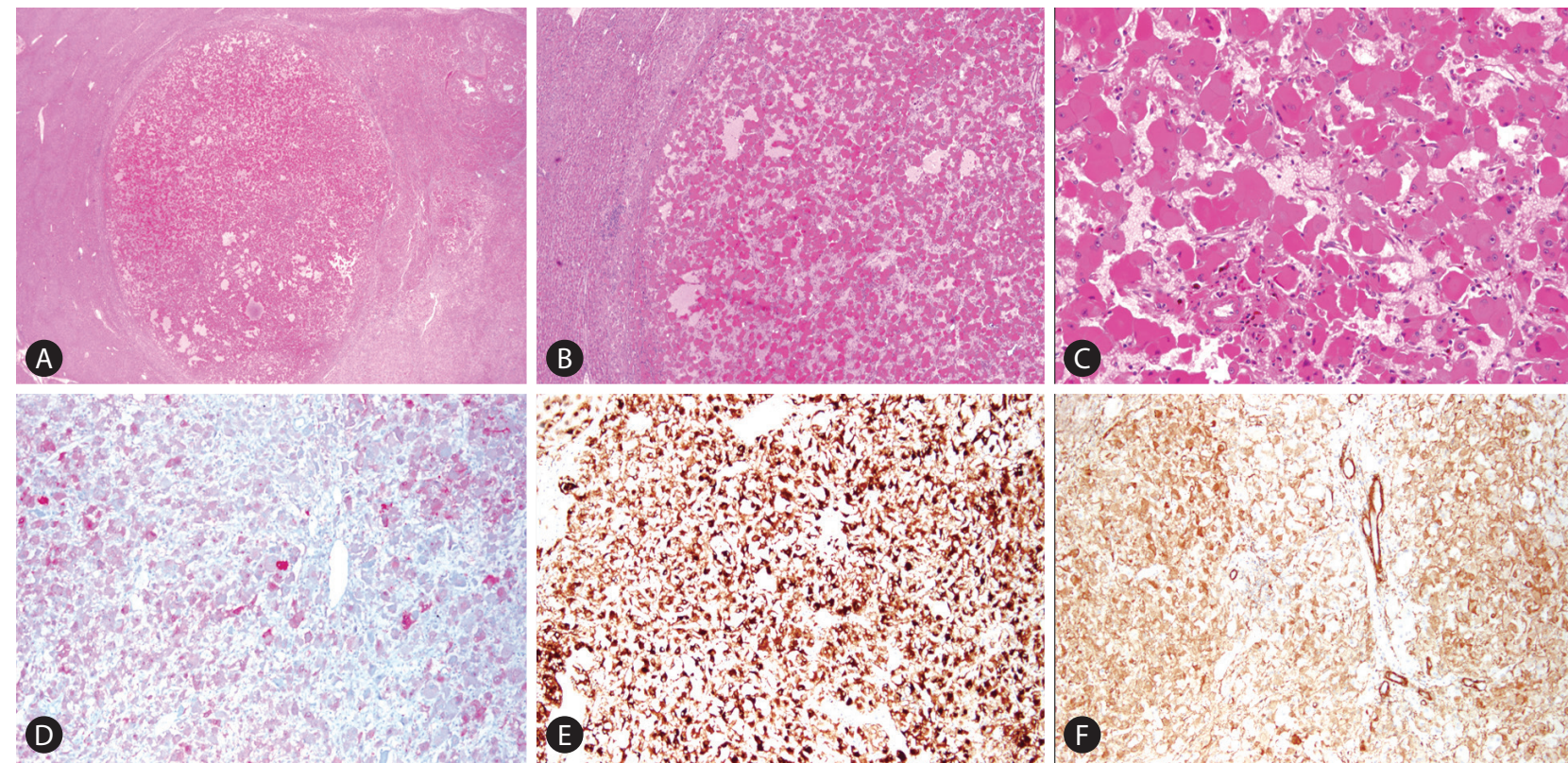

(D)
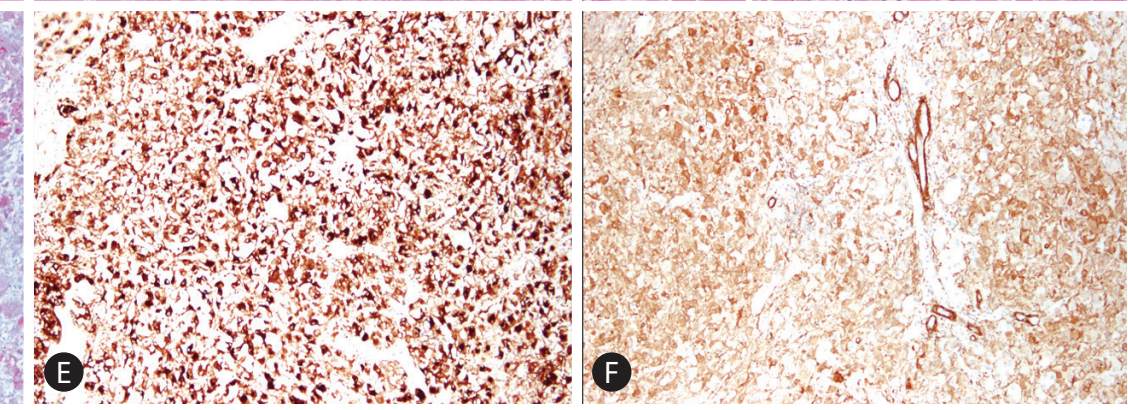

Figure 2. Histopathologic findings of epithelioid angiomyolipoma. (A) Low power view shows well defined mass (H\&E stain, X12.5) (B) Middle power view reveals eosinophilic epithelioid tumor cells. Stromal fibrosis or necrosis is not observed. (H\&E stain, $\times 40$ ) (C) High power picture shows abundant eosinophilic cytoplasm, large and round nuclei, and prominent nucleoli of tumor cells. (H\&E stain, $\times 200$ ) (D-F) Immunohistochemical staining pictures. (D) MelanA, (E) HMB45, and (F) SMA staining is positive in tumor cells (X100). SMA, smooth muscle actin.

tocellular carcinoma, so he underwent left lateral sectionectomy of liver.

On gross examination of liver specimen, the cut surface of liver showed a well-demarcated expanding nodular type mass, measuring $1.2 \times 0.9 \times 0.9 \mathrm{~cm}$ (Fig. 1C). Histologically, the mass was well circumscribed with surrounding normal liver tissue (Fig. 2A). It is consisted of epithelioid cells with abundant eosinophilic granular cytoplasm. The epithelioid cells showed large, round nuclei, and prominent nucleoli with mild pleomorphism (Fig. 2B, C). The vascular component is composed of thin-walled vessels. The adipocyte or smooth muscle cells were not observed. Desmoplastic stroma also was not noted. Tumor necrosis or mitotic figures of tumor cell was not observed. Based on these microscopic, clinical, and radiologic findings, epithelioid angoimyolipoma, epithelioid hemangioendothelioma, adult rhabdomyoma, and heapatocellular carcinoma were considered as differential diagnosis and immunohistochemical stainings were done. HMB45, MelanA, and smooth muscle actin showed diffuse strong positive staining (Fig. 2D, E, F) 
Soo Yeon Lee, et al. Hepatic epithelioid angiomyolipoma

It showed negative staining for anti-hepatocyte, and MyoD1. Based on the histologic findings and results of immunohistochemical staining, the final diagnosis was EAML.

\section{DISCUSSION}

There is no known sex predilection in AML. The tumor is almost sporadic, but its association with tuberous sclerosis complex is revealed in some cases. It classically consists of variable amount of myomatous spindle cell component, thick walled vascular component, and mature adipose tissue component. Some AMLs lack adipose tissue components and only composed of spindle cells with indefinite myomatous differentiation. Nowadays, AMLs are thought as a member of perivascular epithelioid cell tumor (PEComa) family. These PEComa family includes pulmonary lymphangioleiomyoma, and clear cell sugar tumors of various organs. The diagnosis of AML depends on immunohistochemical expression of melanocytic markers HMB45 and MelanA. Myomatous spindle cells also express muscle specific antigen. ${ }^{1,3,5,7}$ EAML is a variant of $A M L$, that consists of predominantly epithelioid cells. EAML is known to have more aggressive behavior ${ }^{8,9}$ and show more association with tuberous sclerosis complex.

$\mathrm{AML}$ in the liver is not infrequently observed and right lobe is more common site for AML than left lobe. The histologic features of hepatic AMLs are similar to AMLs of other organs, that is, mixed variable amounts of myomatous, lipoid, and vascular components. Epithelioid variant of AML (EAML) also occurs in the liver. EAML of the liver is not so widely reported as compared to the EAML of the kidney and soft tissue. In general, EAML shows more frequently poor clinical behavior, it is thought that hepatic EAML will also show more aggressive behavior than classic EAML.

After the first description of Yamasaki et al. ${ }^{2}$ in 2000, 97 cases of hepatic EAML was reported. 1,3,6,10-13 The results are summarized in Table 1. Female predominance was noted in the hepatic EAML (F:M=4.7:1). Reported tumor size was varied from $0.6 \mathrm{~cm}$ to $32 \mathrm{~cm}$. Clinical symptoms were more common in cases with larger tumor. The clinical symptoms were mostly non-specific. In some cases, patients even do not complain any symptoms, and the tumors were found by routine checkup. The most common symptom was abdominal discomfort or pain. The radiologic findings were also non-specific, so that misdiagnosis was common before the pathologic diagnosis. Nearly all tumors were well enhanced in arterial phase because of the high vascularity, and some of them showed decreased enhancement at portal and de-
Table 1. Clinical characteristics of hepatic epithelioid angiomyolipoma

\begin{tabular}{|c|c|c|}
\hline Parameter & & Patients No. (\%) \\
\hline \multirow[t]{2}{*}{ Sex } & Female & $80(82.5)$ \\
\hline & Male & $17(17.5)$ \\
\hline \multirow[t]{2}{*}{ Size } & Min. & $0.6 \mathrm{~cm}$ \\
\hline & Max. & $32 \mathrm{~cm}$ \\
\hline \multirow[t]{7}{*}{ Radiologic diagnosis } & $\mathrm{HCC}$ & $19(19.6)$ \\
\hline & $\mathrm{HA}$ & $8(8.2)$ \\
\hline & $\mathrm{FNH}$ & $2(2.1)$ \\
\hline & AML & $4(4.1)$ \\
\hline & Metastatic tumor & $2(2.1)$ \\
\hline & Hemangioma & $1(1.0)$ \\
\hline & $\mathrm{N} / \mathrm{A}$ & $63(64.9)$ \\
\hline \multirow[t]{3}{*}{ Tuberous sclerosis complex } & Identified & $6(6.2)$ \\
\hline & None & $71(73.2)$ \\
\hline & $\mathrm{N} / \mathrm{A}$ & $20(20.6)$ \\
\hline \multirow[t]{3}{*}{ Recurrence or metastasis } & Identified & $9(9.3)$ \\
\hline & None & $70(72.2)$ \\
\hline & N/A & $18(18.6)$ \\
\hline Total cases & \multicolumn{2}{|r|}{97} \\
\hline
\end{tabular}

N/A, not applicable; HCC, hepatocellular carcinoma; HA, hepatocellular adenoma; FNH, focal nodular hyperplasia; AML, angiomyolipoma.

layed phase. ${ }^{14}$ These findings can mimic the typical radiologic findings of hepatocellular carcinoma.

Gross findings of hepatic EAMLs are usually reported as tan colored nodule with variegated appearance, because of intra-tumoral hemorrhage, adipose component, and in sometimes, necrosis. Microscopic findings of hepatic EAML is characterized by epithelioid tumor cells with plump eosinophilic granular cytoplasm arranged around blood vessesls. Those cells usually show round to polygonal cytoplasm and round and large nucleus with prominent nuclei. In some cases, tumor cells show clear cytoplasm rather than eosinophilic cytoplasm. The adipose component may be scanty or absent. The multinucleated giant cells and large ganglion like tumor cells were common. Usually, mitotic figures of tumor cell is rarely observed. The predominance of epithelioid cell is the most important factor for the diagnosis of EAML. There is still a debate on the cut-off ratio of epithelioid cells needed for the diagnosis of EAML." The immunohistochemical staining is important in the diagnosis of EAML. It is well-known that EAML is posi- 
tive for melanoma markers and smooth muscle marker. Epithelial markers and hepatocyte markers are usually negative. Because hepatocellular carcinoma tumor cells have abundant cytoplasm, hepatic EAMLs are often misdiagnosed as hepatocellular carcinoma without immunohistochemical staining, especially when there is no adipose tissue component.

As shown in Table 1, the association between hepatice EAML and tuberous sclerosis complex seems to be exist. About $6.2 \%$ of reported hepatic EAML patiens had tuberous sclerosis complex. Furthermore, recurrence or metastasis rate of hepatic EAML was reported in considerable range (9.3\%). As though, the recurrence or metastatic rate of hepatic EAML was lower than that of renal EAML, which is reported as about 30\%, hepatic EAML patients should be followed up carefully.

In summary, here we reported a case of hepatic EAML and reviewed the literature. Image findings and pathologic features of EAML can sometimes mimic that of hepatocellular carcinoma, cautions should be paid to avoid misdiagnosis. The association between EAML and tuberous sclerosis complex should be keep in mind. Hepatic EAML sometimes shows aggressive clinical behavior and when the hepatic EAML is diagnosed, careful follow-up is recommended.

\section{Conflicts of Interest}

The authors have no conflicts to disclose.

\section{REFERENCES}

1. Lo RC. Epithelioid angiomyolipoma of the liver: a clinicopathologic study of 5 cases. Ann Diagn Pathol 2013;17:412-415.

2. Yamasaki S, Tanaka S, Fujii H, Matsumoto T, Okuda C, Watanabe G, et al. Monotypic epithelioid angiomyolipoma of the liver. Histopathology 2000;36:451-456

3. Liu W, Meng Z, Liu H, Li W, Wu Q, Zhang X, et al. Hepatic epithelioid angiomyolipoma is a rare and potentially severe but treatable tumor: A report of three cases and review of the literature. Oncol Lett 2016:11:3669-3675.
4. Zhou Y, Chen F, Jiang W, Meng Q, Wang F. Hepatic epithelioid angiomyolipoma with an unusual pathologic appearance: expanding the morphologic spectrum. Int J Clin Exp Pathol 2014;7:6364-6369.

5. Mete 0, van der Kwast TH. Epithelioid angiomyolipoma: a morphologically distinct variant that mimics a variety of intra-abdominal neoplasms. Arch Pathol Lab Med 2011;135:665-670.

6. Fukuda Y, Omiya H, Takami K, Mori K, Kodama Y, Mano M, et al. Malignant hepatic epithelioid angiomyolipoma with recurrence in the lung 7 years after hepatectomy: a case report and literature review. Surg Case Rep 2016;2:31.

7. Xie L, Jessurun J, Manivel JC, Pambuccian SE. Hepatic epithelioid angiomyolipoma with trabecular growth pattern: a mimic of hepatocellular carcinoma on fine needle aspiration cytology. Diagn Cytopathol 2012;40:639-650.

8. Pea M, Bonetti F, Martignoni G, Henske EP, Manfrin E, Colato C, et al. Apparent renal cell carcinomas in tuberous sclerosis are heterogeneous: the identification of malignant epithelioid angiomyolipoma. Am J Surg Pathol 1998;22:180-187.

9. Martignoni G, Pea M, Bonetti F, Zamboni G, Carbonara C, Longa $\mathrm{L}$, et al. Carcinomalike monotypic epithelioid angiomyolipoma in patients without evidence of tuberous sclerosis: a clinicopathologic and genetic study. Am J Surg Pathol 1998;22:663-672.

10. Maebayashi T, Abe K, Aizawa T, Sakaguchi M, Ishibashi N, Abe 0 , et al. Improving recognition of hepatic perivascular epithelioid cell tumor: Case report and literature review. World I Gastroenterol 2015:21:5432-5441.

11. Liu J, Zhang CW, Hong DF, Tao R, Chen Y, Shang MJ, et al. Primary hepatic epithelioid angiomyolipoma: A malignant potential tumor which should be recognized. World I Gastroenterol 2016;22:49084917.

12. Tajima S, Suzuki A, Suzumura K. Ruptured hepatic epithelioid angiomyolipoma: a case report and literature review. Case Rep Oncol 2014;7:369-375.

13. Ortiz S, Tortosa F. Epithelioid angiomyolipoma of the liver: Clinicopathological correlation in a series of 4 cases. Rev Esp Enferm Dig 2016:108:27-30.

14. Ji JS, Lu CY, Wang ZF, Xu M, Song JJ. Epithelioid angiomyolipoma of the liver: CT and MRI features. Abdom Imaging 2013;38:309-314. 